\title{
Progressive Disseminated Histoplasmosis Mimicking as Anal Carcinoma - Look at the Mountain, Not at Stone: A Case Report
}

\author{
Mahaveer Singh' \\ Abhishek Sharma ${ }^{2}$ \\ Kalpana Sankhala ${ }^{3}$ \\ Hemant Bareth $\mathbb{D}^{4}$ \\ Supriya Suman $\mathbb{D}^{4}$
}

'Department of Endocrinology, National Institute of Medical Sciences and Research Hospital, Nims University Rajasthan, Jaipur, India; ${ }^{2}$ Department of General Medicine, National Institute of Medical Sciences and Research Hospital, Nims University Rajasthan, Jaipur, India; ${ }^{3}$ Department of Pathology, National Institute of Medical Sciences and Research Hospital, Nims University Rajasthan, Jaipur, India; ${ }^{4}$ Department of Pharmacy Practice, Nims Institute of Pharmacy, Nims University Rajasthan, Jaipur, India
Correspondence: Mahaveer Singh Department of Endocrinology, National Institute of Medical Sciences and Research Hospital, Nims University, Rajasthan, Jaipur, 303121, India

Tel +918949836075

Email drms.mamcmed@gmail.com

\begin{abstract}
Disseminated histoplasmosis is a systemic disease caused by the dimorphic fungus Histoplasma capsulatum. Here, we are presenting a case of shock who was diagnosed to have primary adrenal insufficiency. This 68-year-old man had bilateral adrenal mass and later presented with chronic fever and ulcerated anal mass in the oncology clinic. The oncologist made a provisional diagnosis of anal carcinoma with adrenal metastasis. $\mathrm{He}$ was suspected of having an adrenal crisis and was admitted to the intensive care unit. He also had granulomatous hepatitis and acute kidney injury. The working diagnosis was changed to systemic inflammatory/infective pathology. The biopsy of the anal tissue done to look for the aetiology showed Histoplasma. He was started on oral itraconazole therapy. He improved symptomatically (resolution of fever, improvement in pain) when assessed after seven days. His anal ulcer healed after 21 days of itraconazole therapy.
\end{abstract}

Keywords: adrenal metastasis, anal carcinoma, Histoplasma, ulcerated anal mass

\section{Background}

Disseminated histoplasmosis is a systemic disease caused by the dimorphic fungus Histoplasma capsulatum. ${ }^{1}$ Histoplasmosis is endemic in the Ohio and Mississippi river valleys, large parts of Latin America and South Asia. ${ }^{2}$ Histoplasmosis is acquired through the inhalation of infectious conidia found in moist, acidic, and nitrogen-rich soil. The clinical severity of histoplasmosis depends on many factors like inhaled quanta, fungal virulence, and host immune response.

In the autopsy series of progressive disseminated histoplasmosis, gastrointestinal symptoms were found in $10 \%$ to $12 \%$ with a higher prevalence of involvement in autopsy series $(20 \%$ to $30 \%){ }^{3}$. The symptoms of gastrointestinal involvement are oral ulceration, dysphagia, odynophagia, diarrhoea, abdominal pain, and weight loss. In progressive disseminated histoplasmosis, anal involvement is infrequent and found in only $2-3 \%$ of patients. ${ }^{3}$ Progressive disseminated histoplasmosis mimicking anal carcinoma is very rare. We are reporting a case of progressive disseminated histoplasmosis, who presented with ulcerated anal mass.

\section{Case Presentation}

Sixty-eight-year-old farmer, case of chronic obstructive pulmonary disease, presented to our outpatient department with fatigue, dizziness, pigmentation, and low blood pressure. He had these symptoms for three months. Suspecting adrenal insufficiency, 
we sent concordant random serum cortisol and plasma adrenocorticotrophic hormone. Low random serum cortisol (3 $\mathrm{ug} / \mathrm{dl})$ and elevated plasma ACTH (120 pg/mL) confirmed the diagnosis of primary adrenal insufficiency. As suspicion of adrenal crisis was there, we admitted him to the intensive care unit. He also had acute kidney injury (serum creatinine $2.3 \mathrm{mg} / \mathrm{dl}$, estimated glomerular filtration rate-30 $\mathrm{mL} / \mathrm{min}$ ) with metabolic acidosis ( $\mathrm{pH}-7.1$, with bicarbonate $11 \mathrm{meq} / \mathrm{l})$ and hyperkalemia (Serum potassium-6 meq/l). He was given a saline bolus to correct the dehydration. Hydrocortisone infusion was given to cover the glucocorticoid replacement. His blood pressure improved along with the recovery of kidney functions. Computed tomography was done after stabilization showed bilateral adrenomegaly (Figure 1), with the size of the right and left adrenals, $3 \times 2 \mathrm{~cm}$ ad $4 \times 3 \mathrm{~cm}$, respectively. Subsequently, the patient had been discharged on $15 \mathrm{mg} / \mathrm{m} 2$ hydrocortisone equivalent and 100 microgram fludrocortisones. We planned for the CT guided adrenal biopsy in the follow-up after ruling out pheochromocytoma.

Two months later, he came to the oncology outpatient clinic with a complaint of pain during defecation and an anal mass with ulceration. Before coming to the oncology clinic, he was evaluated by a surgeon and, suspecting anal carcinoma, was referred back to our institute. The oncologist made a diagnosis of metastatic carcinoma anus with paraneoplastic fever. We were called in to have a look. When we saw him, he looked cachectic and more pigmented than the previous admission. His blood pressure was 90/60 $\mathrm{mmHg}$ in supine position right arm, with severe orthostasis on standing up from bed. His pulse rate was $110 / \mathrm{min}$, and the axillary temperature was 101-degree Fahrenheit. He stopped treatment in between and stopped five days back.

He had a linear ulcer of $4.0 \times 1.5 \mathrm{~cm}$ on the upper anterior gingiva (Figure 2). He had firm hepatomegaly ( $8 \mathrm{~cm}$ below the right subcostal margin). The spleen was enlarged by $6 \mathrm{~cm}$ towards the umbilicus. Chest examination revealed rhonchi and inspiratory crepitations throughout the lung fields.

He had an ulcer on the anal verge, covering 240 degrees on the verge (Figure 3). The ulcer had overhanging edges.

Suspecting adrenal crisis due to treatment noncompliance, he was admitted to the medical intensive care unit. He was given normal saline and hydrocortisone infusion. On investigations, the patient had elevated liver enzymes along with elevated serum alkaline phosphatase. He also had nonoliguric acute kidney injury (Table 1). The ultrasonographic examination revealed hepatosplenomegaly with no focal lesions. Chest X-ray showed prominent broncho-vascular markings. The working diagnosis was changed from the carcinoma anus to a systemic infection/inflammation with granulomatous hepatitis and renal injury.

The differential diagnosis of a patient with an anal ulcer, oral ulcer, primary adrenal insufficiency, granulomatous

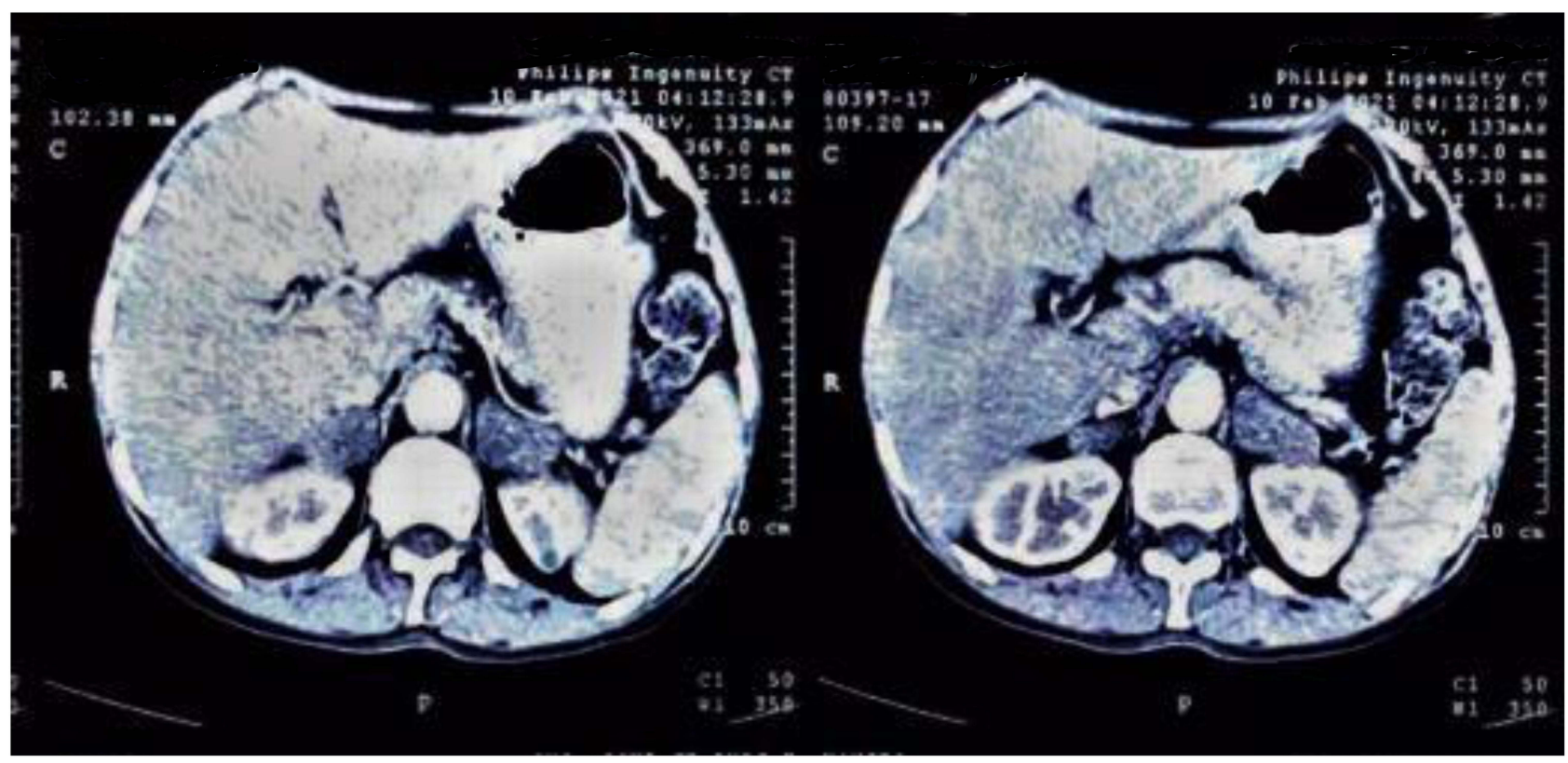

Figure I CECT abdomen shows bilateral adrenal enlargement with the right $(3 \times 2 \mathrm{~cm})$ and left $(4 \times 3 \mathrm{~cm})$. Both the adrenals were heterogeneous, had irregular margins and calcifications. 


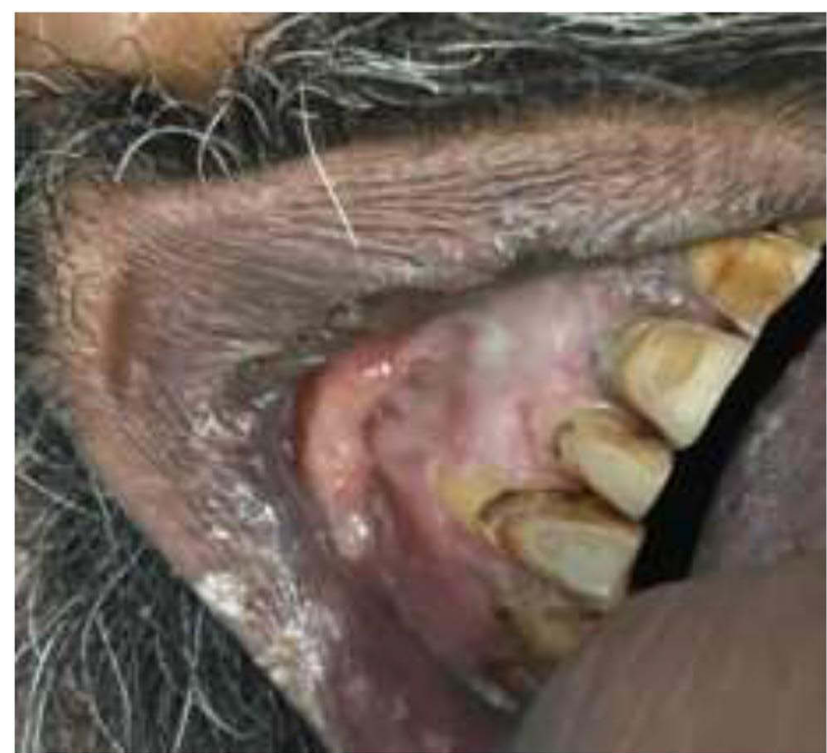

Figure $24 \mathrm{~cm} \times 1.5 \mathrm{~cm}$ ulcer on the upper gum line, with a whitish coating over the ulcer.

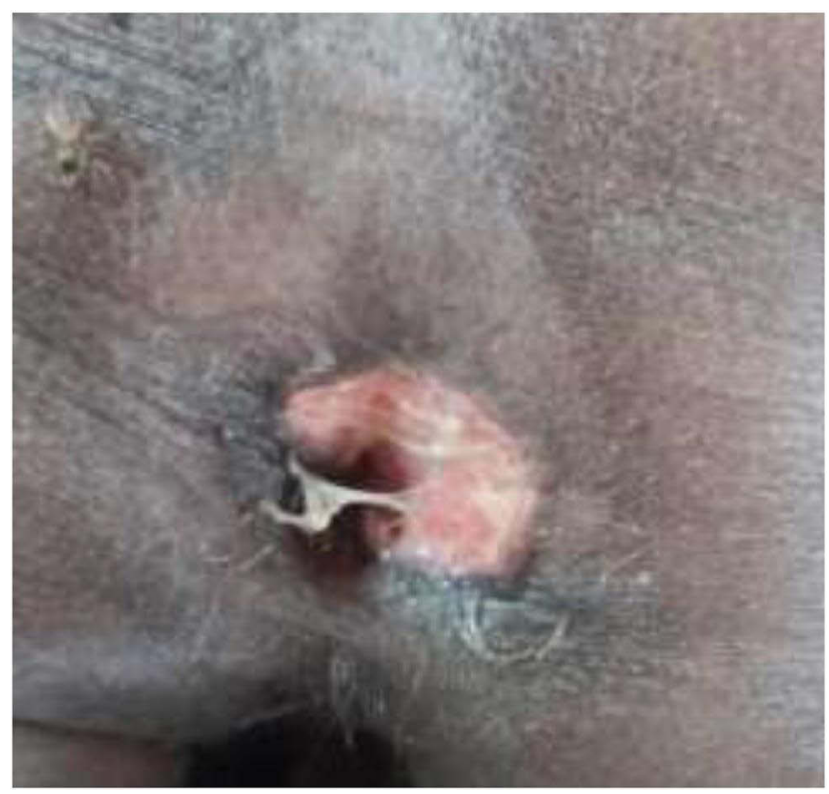

Figure 3 Anal region showing a $2.0 \times 2.0-\mathrm{cm}$ area of induration at the left lateral anus.

hepatitis, and kidney injury are disseminated tuberculosis, disseminated fungal infection (histoplasmosis), disseminated CMV infection and syphilis.

The patient was stabilized on the hydrocortisone infusion with a plan to biopsy the anal ulcer. The adrenal biopsy would have been the subsequent investigation if the diagnosis was not confirmed on the anal biopsy. Anal biopsy revealed focally ulcerated squamous mucosa. Underlying stroma showed neutrophils, lymphocytes, plasma cells and macrophages with budding oval yeast forms surrounded by a halo suggesting the diagnosis of Histoplasma capsulatum. The Histoplasma capsulatum was confirmed by PAS positivity (Figure 4). As the diagnosis was established on the anal biopsy and clinically fit into the progressive disseminated histoplasmosis, we kept the adrenal biopsy as a salvage procedure. The nephrologist was in the opinion of wait and watch for the kidney injury as it may improve with the treatment of underlying disease.

The patient was started on oral itraconazole therapy (800 mg a day for three days followed by $200 \mathrm{mg}$ twice a day). He was discharged on $15 \mathrm{mg} / \mathrm{m} 2$ of hydrocortisone as a steroid replacement, itraconazole (200 $\mathrm{mg}$ twice a day with milk) as an antifungal treatment. The patient was advised to monitor the temperature every six hours and come if any adverse effects. We planned weekly follow-up in the first month to assess the clinical response (resolution of the fever, healing of the ulcers, improvement in kidney injury), steroid replacement (under or over replacement) and treatment side effects (gastritis, hepatitis). The imaging of the adrenals and cortisol reserve will be tested after six months of therapy.

At seven days follow-up, the patient's appetite improved, and he became afebrile.

His renal function and liver function improved when assessed on day seven compared with the status at discharge. The comparison is shown in Table 1. His pain during defecation also decreased.

His fatigue improved, and he gained $2 \mathrm{~kg}$ of weight after two weeks. The anal ulcer healed after three weeks of antifungal therapy.

\section{Patient's Perspective}

I was terrified when the suspicion of anal carcinoma was made and discussed with me. I had fever and anorexia for a long time, causing a severe dent in my quality of life. My doctor told me that it could be a systemic disease in the background of the bilateral adrenal enlargement and primary adrenal insufficiency, with granulomatous hepatitis and acute kidney injury. I was advised for a biopsy from the anal ulcer. I got relieved when I heard that it is not cancer and is a fungal infection. The happiness increased further when during treatment discussions, I was informed that it is curable.

\section{Discussion}

Histoplasma is a fungus of the eumycete's family. Histoplasmosis capsulatum var capsulatum is the most 
Table I Lab Investigations at Admission, at Discharge (*Day Eight After Admission and Three Days After Itraconazole Therapy) and Follow UP at Day 7 in the Endocrine OPD

\begin{tabular}{|c|c|c|c|c|}
\hline Investigation & At Admission & At Discharge* & $\begin{array}{l}\text { At First, Follow } \\
\text { Up, Seven Days } \\
\text { After Discharge }\end{array}$ & Normal Range \\
\hline \multicolumn{5}{|l|}{ Hematology Analysis Report } \\
\hline $\mathrm{Hb}(\mathrm{g} / \mathrm{dl})$ & 10.1 & & 11 & $13-18$ \\
\hline Total leucocyte count (ths/ul) & 7.8 & & 7000 & $4.5-12.5$ \\
\hline Blood neutrophils (\%) & 72 & & 70 & $45-75$ \\
\hline Blood lymphocytes (\%) & 22 & & 24 & $25-45$ \\
\hline Blood monocytes (\%) & 04 & & 4 & $2-8$ \\
\hline Blood eosinophils (\%) & 02 & & 2 & $\mathrm{I}-6$ \\
\hline Platelet Count $\left(\times 10^{3} / \mathrm{uL}\right)$ & 121 & & 150 & $150-450$ \\
\hline \multicolumn{5}{|l|}{ Renal Function Tests } \\
\hline Serum Urea (mg/dl) & 69 & 51 & 71 & $15-45$ \\
\hline Serum Creatinine (mg/dl) & 2.51 & 1.90 & 1.3 & $0.6-1.6$ \\
\hline Serum Calcium (mg/dl) & 7.1 & & 7.5 & $8.5-10.5$ \\
\hline Serum phosphorus-Inorg (mg/dl) & 3.6 & & 3.5 & $2.5-5$ \\
\hline Serum sodium $(\mathrm{mmol} / \mathrm{L})$ & 139 & 136 & 136 & $135-145$ \\
\hline Serum potassium (mmol/L) & 2.9 & 3.0 & 3.95 & $3.5-5.5$ \\
\hline Urine spot potassium (meq/l) & $40 \mathrm{meq} / \mathrm{l}$ & & & $25-125$ \\
\hline \multicolumn{5}{|c|}{ Liver function tests } \\
\hline Serum SGOT (IU/L) & 119 & 59 & 37 & $10-50$ \\
\hline Serum SGPT (IU/L) & 99 & 64 & 93 & $14-63$ \\
\hline Serum albumin $(g / d l)$ & 3.0 & 2.4 & 2.6 & $3.5-5.2$ \\
\hline Serum globulin $(g / d l)$ & 3.3 & 4.0 & 4.1 & $2.3-3.6$ \\
\hline Serum alkaline phosphatase (iu/l) & 1174 & 482 & 314 & $45-145$ \\
\hline Serum bilirubin $(T / D) \mathrm{mg} / \mathrm{dl}$ & $0.6 / 0.3$ & $0.6 / 0.3$ & $0.5 / 0.2$ & $0.0-1.2 / 0.0-0.25$ \\
\hline
\end{tabular}

prevalent species of Histoplasma. Histoplasmosis is also called "Darling's disease", "Spelunker's disease", or "caves disease". 4,5 In India, histoplasmosis is endemic in the Gangetic belt of the UP and West Bengal. ${ }^{6}$

Histoplasma resides in its microfoci (droppings, bird's feathers, soil of old buildings, old bushes, and trees). Disruption of these microfoci causes the spread of microconidia in the air and inhalation into the lung. Dissemination is not very common and occurs in $0.1 \%$ to $1 \%$ of patients. Some of these patients with dissemination develop progressive disseminated histoplasmosis. ${ }^{7}$

Gastrointestinal involvement was found in $20 \%$ to $30 \%$ of cases of progressive disseminated histoplasmosis. ${ }^{8}$ In a review of the 77 reported cases of gastrointestinal histoplasmosis, the small intestine was the most commonly involved site. Ileal ulceration was the most common symptom of gastrointestinal histoplasmosis (Table 2). In histopathology analysis of gastrointestinal lesions, Lamps et al evaluated 56 specimens from 52 patients evaluated with hematoxylin and eosin and silver stain and found that in gross gastrointestinal features, ulcers (49\% of patients) were the most common clinical manifestation of gastrointestinal histoplasmosis, followed by nodules $(21 \%)$, haemorrhage $(13 \%)$, obstructive masses $(6 \%)$. Lymphohistiocytic infiltration $(83 \%)$ was the most common finding on microscopic examination.

The first description of anorectal histoplasmosis was done by Weiss et al in 1952. ${ }^{9}$ They described a 62-year-old man who had dysphagia, weight loss, fever, and pain during defecation. Proctoscopy of the patient showed polyp in the rectum and anal canal. Biopsy of the anal polyp showed Histoplasma.

In another case report, similar to our case, Matthew

Wheel wright reported a 66-year-old male patient who presented with an anal ulcer with suspicion of the carcinoma on imaging. ${ }^{10}$ Core biopsy of anal mass showed intracellular yeast suggestive of Histoplasma. 


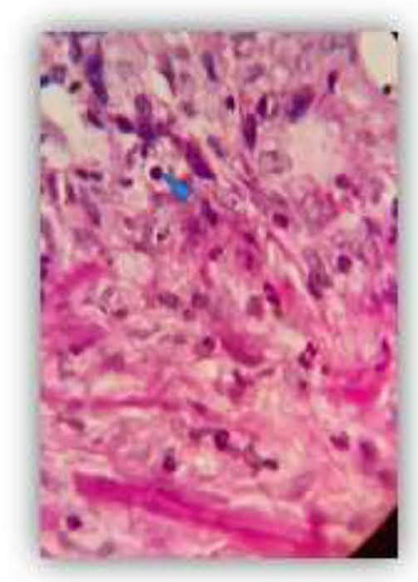

A

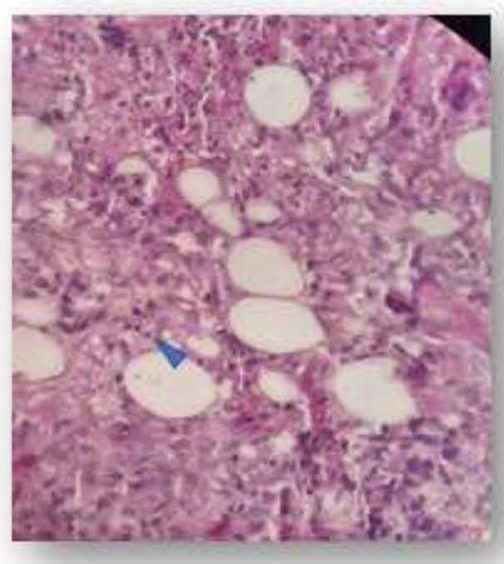

B

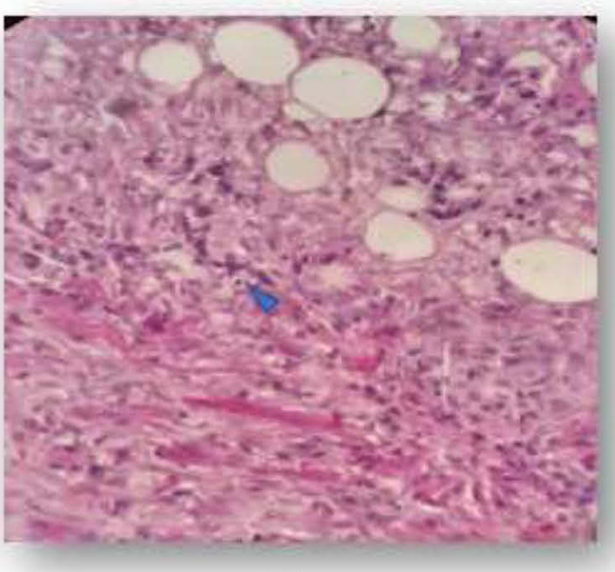

C

Figure 4 Histologic features of perianal biopsy: (A) High power (1000x) of H\&E-stained section demonstrates yeast of Histoplasma capsulatum; (B) High power (I000x) of PAS-stained section demonstrates a mononuclear inflammatory infiltrate composed of histiocytes, lymphocytes, and plasma cells along with Histoplasma capsulatum; (C) Low power $(400 \times)$ of PAS-stained section shows histiocytes with intracytoplasmic fungal yeast forms $(2-5 \mu m)$ with a characteristic halo.

Anorectal histoplasmosis can present with lower intestinal bleeding. Mullick et al reported a case in which a 69-year-old man presented with lower gastrointestinal bleeding. ${ }^{11}$ The colonoscopy showed an anal mass mimicking squamous cell carcinoma on appearance. Biopsy of this mass showed Histoplasma on the periodic acid Schiff and Gomori methenamine silver stains.

Antifungal agent itraconazole or amphotericin B can be used for the treatment of adrenal histoplasmosis. The clinical phenotype of the adrenal histoplasmosis determines the treatment protocol. Moderate to severe gastrointestinal histoplasmosis requires the amphotericin B treatment for initial 2 to 4 weeks, followed by itraconazole for 1 to 2 years. Mild gastrointestinal progressive disseminated histoplasmosis can be treated by oral itraconazole therapy for 1 to 2 years. ${ }^{12}$ Mortality in disseminated histoplasmosis is $80 \%$ to $100 \%$ without treatment and $25 \%$ with treatment. ${ }^{13}$ Relapse has been found in $10 \%$ to $20 \%$ of immunocompetent patients after nine years of follow-up. In AIDS, the relapse rate is very high, approaching $80 \%$ if the patient's CD4 count is persistently below $100 /$ ul. $^{1,14}$

\section{Conclusion}

The exophytic reactive ulcerative growth of the anal canal can mimic carcinoma anus. In our case, systemic symptoms like fever, anorexia, and multiorgan dysfunction point towards infective causes like progressive disseminated histoplasmosis. Our case illustrates that the bull's eye

Table 2 Clinicopathologic Manifestations of Gastrointestinal Histoplasmosis

\begin{tabular}{|l|l|l|l|}
\hline Site & Frequency of Involvement & Clinicopathologic Manifestation & Frequency \\
\hline Small Intestine & $56-79 \%$ (ileum most common) & Ulceration (ileum most common) & $30-49 \%$ \\
\hline Large Intestine & $55-65 \%$ & Mucosal nodules & $21 \%$ \\
\hline Stomach & $14-17 \%$ & Stricture or mass & $18 \%$ \\
\hline Esophagus & $8-18 \%$ & Lymphoid hyperplasia & $15 \%$ \\
\hline Rectum & $13 \%$ & Hemorrhage or petechiae & $13 \%$ \\
\hline Pancreas & $6 \%$ & Mass & $6 \%$ \\
\hline Gallbladder & $6 \%$ & Perforation & $5.2 \%$ \\
\hline Anus & $2 \%$ & Ulceration & \\
\hline Appendix & $2-2.5 \%$ & - & \\
\hline
\end{tabular}


approach, used by today's specialist era, can miss the whole clinical picture. This patient also reminds us of the title "By looking at stone, a mountain can be missed".

\section{Abbreviations}

$\mathrm{CMV}$, cytomegalovirus; ACTH, adrenocorticotropic hormone; CT, computed tomography; AmB, amphotericin B; CD4, cluster of differentiation; PAS, periodic acid-Schiff.

\section{Data Sharing Statement}

All data generated during this study are available from the corresponding author on reasonable request.

\section{Patient Consent}

Patient provided written informed consent for the case details and accompanying images to be published. No institutional approval was required to publish this case report.

\section{Author Contributions}

All authors made a significant contribution to the work reported, whether that is in the conception, study design, execution, acquisition of data, analysis and interpretation, or in all these areas; took part in drafting, revising or critically reviewing the article; gave final approval of the version to be published; have agreed on the journal to which the article has been submitted; and agree to be accountable for all aspects of the work.

\section{Funding}

No funding was received for this case report.

\section{Disclosure}

The authors report no conflicts of interest.

\section{References}

1. Wheat LJ. Histoplasmosis: a review for clinicians from non-endemic areas. Mycoses. 2006;49(4):274-282. doi:10.1111/j.1439-0507.2006. 01253.x

2. Bahr NC, Antinori S, Wheat LJ, Sarosi GA. Histoplasmosis infections worldwide: thinking outside of the Ohio River valley. Curr Trop Med Rep. 2015;2(2):70-80. doi:10.1007/s40475-015-00 44-0

3. Lamps LW, Molina CP, West AB, Haggitt RC, Scott MA. The pathologic spectrum of gastrointestinal and hepatic histoplasmosis. Am J Clin Pathol. 2000;113(1):64-72. doi:10.1309/X0Y2-P3GYTWE8-DM02

4. Pan B, Chen M, Pan W, Liao W. Histoplasmosis: a new endemic fungal infection in China? Review and analysis of cases. Mycoses. 2013;56(3):212-221. doi:10.1111/myc.12029

5. Panackal AA, Hajjeh RA, Cetron MS, Warnock DW. Fungal infections among returning travelers. Clin Infect Dis. 2002;35 (9):1088-1095. doi:10.1086/344061

6. Randhawa HS. Occurrence of histoplasmosis in Asia. Mycopathol Mycol Appl. 1970;41:75-89. doi:10.1007/BF02051485

7. Hage CA, Azar MM, Bahr N, et al. Histoplasmosis: up-to-date evidence-based approach to diagnosis and management. Semin Respir Crit Care Med. 2015;36:729-745. doi:10.1055/s-00351562899

8. Cappell MS, Mandell W, Grimes MM, et al. Gastro-intestinal histoplasmosis. Dig Dis Sci. 1988;33:353-360. doi:10.1007/ BF01535762

9. Weiss ED, Haskell BF. Anorectal manifestations of histoplasmosis. Am J Surg. 1952;84:541-544. doi:10.1016/0002-9610(52)90083-4

10. Wheelwright M, Yousaf H, Plummer R, et al. Perianal histoplasmosis presenting as a mass suspicious for malignancy: a case report with review of gastrointestinal manifestations of histoplasmosis. Am J Case Rep. 2019;20:1740-1744. doi:10.12659/AJCR.918220

11. Mullick SS, Mody DR, Schwartz MR. Cytology of gastrointestinal histoplasmosis: a report of two cases with differential diagnosis and diagnostic pitfalls. Acta Cytol. 1996;40:989-994. doi:10.1159/ 000334015

12. Wheat LJ, Freifeld AG, Kleiman MB, et al. Clinical practice guidelines for the management of patients with histoplasmosis: 2007 update by the Infectious Diseases Society of America. Clin Infect Dis. 2007;45:807-825. doi:10.1086/521259

13. Rubin H, Furcolow ML, Yates JL, et al. The course and prognosis of histoplasmosis. Am J Med. 1959;27:278-288. doi:10.1016/00029343(59)90347-X

14. Wheat J. Endemic mycoses in AIDS: a clinical review. Clin Microbiol Rev. 1995;8:146-159. doi:10.1128/cmr.8.1.146-159. 1995

\section{Publish your work in this journal}

The Journal of Multidisciplinary Healthcare is an international, peerreviewed open-access journal that aims to represent and publish research in healthcare areas delivered by practitioners of different disciplines. This includes studies and reviews conducted by multidisciplinary teams as well as research which evaluates the results or conduct of such teams or healthcare processes in general. The journal covers a very wide range of areas and welcomes submissions from practitioners at all levels, from all over the world. The manuscript management system is completely online and includes a very quick and fair peer-review system. Visit http://www.dovepress.com/testimonials. php to read real quotes from published authors. 